\title{
Commentary on "C-Reactive Protein for the Diagnosis of Late-Onset Infections in Newborn Infants"
}

\author{
Mohan Pammi ${ }^{a}$ Prakesh S. Shah ${ }^{b}$ \\ aBaylor College of Medicine, Houston, TX, USA; ${ }^{b}$ Mount Sinai Hospital and Institute of HPME, University of Toronto, \\ Toronto, ON, Canada
}

\section{Commentary}

Assessing diagnostic tests for accuracy and reliability is an important component of evidence-based practice. The Cochrane Screening and Diagnostic Test Methods group (https://methods.cochrane.org/sdt/welcome) oversees the editorial process for systematic reviews of diagnostic test accuracy. Brown et al. [1] have published a Cochrane diagnostic test accuracy review on the accuracy of C-reactive protein (CRP) in the diagnosis of late-onset infection in newborn infants. Preterm birth and infections are the most common causes of neonatal deaths worldwide [2]. Early diagnosis and treatment of infections can reduce neonatal mortality and morbidity. Late-onset infection, defined as occurring after $48-72 \mathrm{~h}$ of age, occurs in between 10 and $40 \%$ of neonates depending upon population studied [3, 4]. Risk factors include lower gestational age, higher severity of illness at birth, the presence of central catheters for more than 4 days, receipt of parenteral nutrition for more than 1 week, and longer duration without enteral feeds [5]. The gold standard for the diagnosis of late-onset infection is isolation of pathogenic organisms from culture of the blood, cerebrospinal fluid, or other sterile body fluids; however, the culture results can take up to about $24-48 \mathrm{~h}$ after incubation. The sensitivity of blood culture is often questioned given the low colony counts $(<4$ colony form-

karger@karger.com

(C) 2021 S. Karger AG, Basel

www.karger.com/neo

Karger! ing units/mL of blood) in neonatal infection compared to higher colony counts in adults and children [6]. Low accuracy of blood cultures can result from non-sterile techniques of blood collection (contamination) or low blood volume inoculation $(<1 \mathrm{~mL}$ of blood) [7]. Alternative methods of detection of infection including molecular markers of infection (microbial DNA by PCR or sequencing) [8] or inflammatory biomarkers such as CRP, procalcitonin, and cytokines/chemokines may have diagnostic potential. These diagnostic markers may be used for screening high-risk neonates, early ruling in or ruling out infection, or in following their trend in case of evolving infection and in response to therapy $[9,10]$.

Brown and colleagues [1] systematically reviewed the diagnostic accuracy of CRP (index test) in the initial evaluation of sepsis in newborn infants and compared it to blood culture (reference standard). They included cohort and cross-sectional studies, which evaluated CRP in the initial work up of late-onset infection. Included studies defined "late-onset" as infection that occurred from after $48 \mathrm{~h}$ of age and up to 6 days of age, although most studies defined it as after $72 \mathrm{~h}$ of age. Second, they included 20 studies (1,615 newborn infants), which were mostly small, singlecenter cohort studies (sample size ranging from 11 to 184 infants, with 4 studies having a sample size less than 25). Sixteen of the 20 studies enrolled preterm infants predom- 


\begin{tabular}{|c|c|c|c|c|c|c|c|c|c|c|c|}
\hline Study & TP & $\mathrm{FP}$ & FN & $\mathrm{TN}$ & $\begin{array}{l}\text { Predefined } \\
\text { threshold }\end{array}$ & $\begin{array}{l}\text { Standard } \\
\text { threshold } \\
(5-10 \mathrm{mg} / \mathrm{L})\end{array}$ & $\begin{array}{l}\text { Sensitivity } \\
(95 \% \mathrm{Cl})\end{array}$ & $\begin{array}{l}\text { Specificity } \\
(95 \% \mathrm{Cl})\end{array}$ & $\begin{array}{c}\text { Sensitivity } \\
(95 \% \mathrm{Cl})\end{array}$ & & $\begin{array}{c}\text { Specificity } \\
(95 \% \mathrm{Cl})\end{array}$ \\
\hline Aminullah, 2001 & 4 & 1 & 3 & 3 & yes & no & $0.57(0.18,0.90)$ & $0.75(0.19,0.99)$ & $\longrightarrow$ & & $\longrightarrow$ \\
\hline Benitz, 1998 & 33 & 41 & 20 & 90 & no & yes & $0.62(0.48,0.75)$ & $0.69(0.60,0.77)$ & $\longrightarrow-$ & & $\rightarrow-$ \\
\hline Bohnhorst, 2012 & 40 & 18 & 18 & 94 & yes & yes & $0.69(0.55,0.80)$ & $0.84(0.76,0.90)$ & $\rightarrow$ & & $\rightarrow$ \\
\hline Boo, 2008 & 10 & 7 & 7 & 62 & yes & no & $0.59(0.33,0.82)$ & $0.90(0.80,0.96)$ & $\longrightarrow$ & & $\rightarrow$ \\
\hline Bustos, 2012 & 3 & 1 & 22 & 27 & no & no & $0.12(0.03,0.31)$ & $0.96(0.82,1.00)$ & $\rightarrow-$ & & $\longrightarrow$ \\
\hline Chan, 1997 & 14 & 26 & 16 & 96 & yes & yes & $0.47(0.28,0.66)$ & $0.79(0.70,0.86)$ & $\longrightarrow$ & & - \\
\hline Choo, 2012 & 1 & 2 & 6 & 3 & yes & yes & $0.14(0.00,0.58)$ & $0.60(0.15,0.95)$ & $\rightarrow-$ & & $\rightarrow$ \\
\hline Decembrino, 2015 & 4 & 11 & 4 & 22 & no & yes & $0.50(0.16,0.84)$ & $0.67(0.48,0.82)$ & 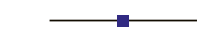 & & $\longrightarrow$ \\
\hline Doellner, 1998 & 5 & 6 & 2 & 9 & yes & yes & $0.71(0.29,0.96)$ & $0.60(0.32,0.84)$ & $\longrightarrow$ & & $\longrightarrow$ \\
\hline Fendler, 2008 & 48 & 3 & 16 & 11 & no & no & $0.75(0.63,0.85)$ & $0.79(0.49,0.95)$ & $\rightarrow$ & & $\longrightarrow$ \\
\hline Hisamuddin, 2015 & 11 & 9 & 27 & 9 & yes & yes & $0.29(0.15,0.46)$ & $0.50(0.26,0.74)$ & $\longrightarrow$ & & $\longrightarrow$ \\
\hline Jacquot, 2009 & 17 & 6 & 13 & 37 & yes & yes & $0.57(0.37,0.75)$ & $0.86(0.72,0.95)$ & $\longrightarrow$ & & $\rightarrow-$ \\
\hline Kipfmueller, 2015 & 3 & 3 & 4 & 15 & yes & yes & $0.43(0.10,0.82)$ & $0.83(0.59,0.96)$ & & & $\longrightarrow$ \\
\hline Kordek, 2014 & 23 & 5 & 11 & 13 & yes & yes & $0.68(0.49,0.83)$ & $0.72(0.47,0.90)$ & $\longrightarrow$ & & $\longrightarrow$ \\
\hline Kumar, 2010 & 55 & 60 & 1 & 26 & yes & yes & $0.98(0.90,1.00)$ & $0.30(0.21,0.41)$ & & $\rightarrow$ & $\rightarrow-$ \\
\hline $\mathrm{Ng}, 1997$ & 27 & 2 & 18 & 54 & yes & yes & $0.60(0.44,0.74)$ & $0.96(0.88,1.00)$ & $\longrightarrow-$ & & $\rightarrow$ \\
\hline Pynn, 2015 & 30 & 35 & 7 & 67 & yes & yes & $0.81(0.65,0.92)$ & $0.66(0.56,0.75)$ & $\longrightarrow$ & & $\rightarrow$ \\
\hline Seibert, 1990 & 13 & 30 & 10 & 47 & yes & yes & $0.57(0.34,0.77)$ & $0.61(0.49,0.72)$ & $\longrightarrow$ & & $\rightarrow$ \\
\hline Sherwin, 2008 & 14 & 1 & 20 & 17 & no & no & $0.41(0.25,0.59)$ & $0.94(0.73,1.00)$ & $\longrightarrow$ & & $\longrightarrow$ \\
\hline Verboon-Maciolek, 2006 & 24 & 14 & 13 & 15 & no & no & $0.65(0.47,0.80)$ & $0.52(0.33,0.71)$ & $\longrightarrow$ & & $\longrightarrow$ \\
\hline & & & & & & & & & $\begin{array}{lllllll} & & & & & 1 & 1 \\
0 & 0.2 & 0.4 & 0.6 & 0.8\end{array}$ & $1 \longdiv { 1 }$ & 0.20 .40 .60 .8 \\
\hline
\end{tabular}

Fig. 1. Forest plot of sensitivity and specificity of CRP in late-onset sepsis in newborn infants. The figure shows the estimated sensitivity and specificity of the study (square) and its 95\% CI (horizontal line). CRP, C-reactive protein; CI, confidence interval.

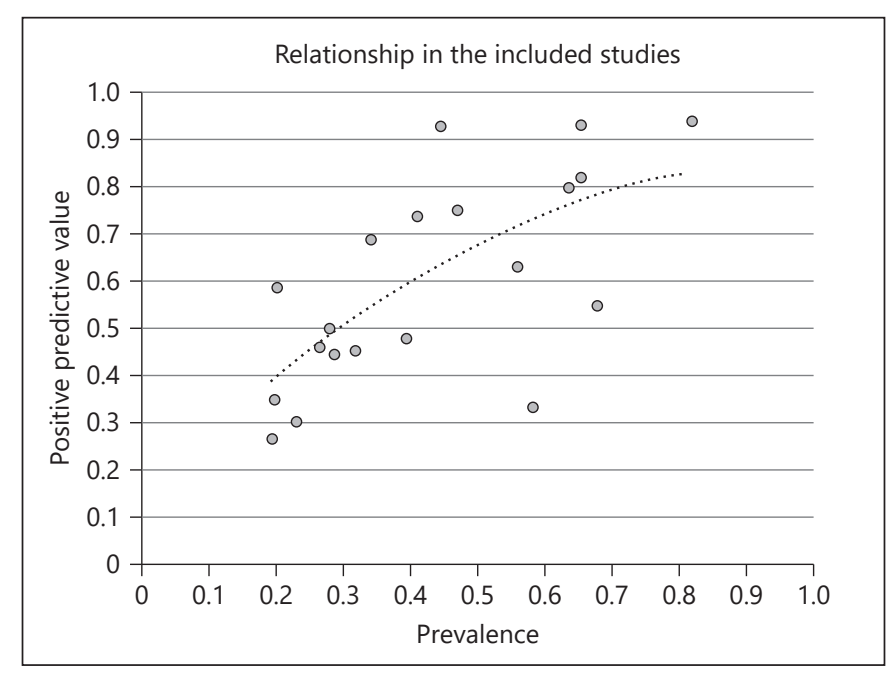

Fig. 2. Correlation of positive predictive values with the prevalence of late-onset sepsis in the studies included in the review.

inantly. Third, the prevalence of late-onset infection in the included studies ranged from 20 to $82 \%$ with a median rate of $40 \%$. Finally, most studies ( 14 of 20 ) reported a prespecified threshold or cutoff ranging from 1 to $12 \mathrm{mg} / \mathrm{L}(12 / 14$ used a cutoff between 5 and $10 \mathrm{mg} / \mathrm{L}$ ) for diagnosis of in- fection, whereas 6 studies did not define a cutoff a priori. The risk of bias as assessed using QUADAS- 2 tool, as recommended by the Cochrane screening and diagnostic test methods group, was reported to be low in the included studies, and the evidence was rated as moderate quality (serious issue with heterogeneity) [11]. The authors were unable to perform subgroup analysis by gestational age or by type of microorganism due to lack of sufficient data.

The extracted data from the studies were synthesized in a meta-analysis using the hierarchical summary receiver operating curve (HSROC model), which incorporates the varying thresholds of the index test used in different studies [12]. Key finding in this meta-analysis was the low sensitivity of CRP (Fig. 1); at median reported specificity of 0.74 , the sensitivity was 0.62 (95\% CI $0.50-0.73$ ); at the lower quartile reported specificity of 0.61 , the sensitivity was 0.76 (95\% CI 0.65-0.84); and at the upper quartile reported specificity of 0.85 , the sensitivity was 0.44 ( $95 \%$ CI $0.32-0.57$ ).

Clinical heterogeneity including the prevalence of confirmed infection is a limitation to the generalizability of the findings of the review. Positive predictive values of a diagnostic test vary with the prevalence of the target condition, and the correlation of positive predictive value with prevalence of infection from the studies included in this review is depicted in Figure 2. If the summary sensitivity (0.62) at 
median specificity $(0.74)$ was applied to a hypothetical cohort of 1,000 newborn infants with a prevalence of lateonset infection of $40 \%$, we would miss 156 cases of sepsis (false negatives) and wrongly diagnose 159 infants with sepsis (false positives). If the prevalence were $10 \%$, we would miss 38 cases of sepsis and wrongly diagnose 234 infants with sepsis. Independent of the prevalence, singletime CRP testing at the initial diagnosis of sepsis has lower diagnostic accuracy than would be useful in practice.

This review does not address serial monitoring of CRP for diagnosis of infection or to follow response after therapy. The reason for the false positives could be elevation of CRP in noninfective inflammatory conditions and false negatives could be because of the time lag in elevation of CRP after infection. Research on alternative biomarkers that are not elevated in noninfective conditions but rapidly increase after infection without a significant time lag is necessary and may have higher diagnostic accuracy in neonatal sepsis [10]. Advances in next-generation sequencing methods have improved detection of infection based on microbial nucleic acid. Molecular microbiological methods may provide rapid identification of the infecting organism and antimicrobial susceptibility patterns, which may be promising alternatives to CRP or other inflammatory biomarkers [8].

\section{Acknowledgement}

Cochrane Neonatal Reviews are produced with support from Vermont Oxford Network, a worldwide collaboration of health professionals dedicated to providing evidence-based care of the highest quality for newborn infants and their families.

\section{Conflict of Interest Statement}

The authors serve in the editorial group of the Cochrane Neonatal.

\section{Funding Sources}

M.P. is funded by NIH grants, R03HD098482 and R21HD091718, not related to this commentary, and the funders had no role in this manuscript. P.S.S. is supported by an Applied Research Chair in Reproductive and Child Health Services and Policy Research from the Canadian Institutes of Health Research.

\section{Author Contributions}

M.P. wrote the initial draft of the manuscript and made final revisions. P.S. provided intellectual input, produced Figure 2, and approved the final version of the manuscript.

\section{References}

1 Brown JVE, Meader N, Cleminson J, McGuire W. C-reactive protein for diagnosing late-onset infection in newborn infants. Cochrane Database Syst Rev. 2019 Jan 14;1(1): CD012126.

2 Liu L, Oza S, Hogan D, Chu Y, Perin J, Zhu J, et al. Global, regional, and national causes of under-5 mortality in 2000-15: an updated systematic analysis with implications for the Sustainable Development Goals. Lancet. 2016 Dec 17;388(10063):3027-35.

3 Shah P, Yoon EW, Chan P, Committee MARR. The report of the Canadian Pediatric Society; 2014 : https://www.canadianneonatalnetwork.org/Portal/LinkClick.aspx?filetic ket=eGgxmMubxjk\%3D\&tabid=39.

4 Shane AL, Stoll BJ. Neonatal sepsis: progress towards improved outcomes. J Infect. 2014 Jan;68(Suppl 1):S24-32..
5 Shah J, Jefferies AL, Yoon EW, Lee SK, Shah PS. Risk factors and outcomes of late-onset bacterial sepsis in preterm neonates born at $<32$ weeks' gestation. Am J Perinatol. 2015 Jun;32(7):675-82.

6 Schelonka RL, Chai MK, Yoder BA, Hensley D, Brockett RM, Ascher DP. Volume of blood required to detect common neonatal pathogens. J Pediatr. 1996 Aug;129(2):275-8..

7 Isaacman DJ, Karasic RB, Reynolds EA, Kost SI. Effect of number of blood cultures and volume of blood on detection of bacteremia in children. J Pediatr. 1996 Feb;128(2):190-5..

8 Pammi M, Flores A, Versalovic J, Leeflang MM. Molecular assays for the diagnosis of sepsis in neonates. Cochrane Database Syst Rev. 2017 Feb 25;2(2):CD011926..
9 Bossuyt PM, Irwig L, Craig J, Glasziou P. Comparative accuracy: assessing new tests against existing diagnostic pathways. BMJ. 2006 May 6;332(7549):1089-92..

10 Hedegaard SS, Wisborg K, Hvas AM. Diagnostic utility of biomarkers for neonatal sepsis: a systematic review. Infect Dis. 2015 Mar; 47(3):117-24..

11 Whiting PF, Rutjes AW, Westwood ME, Mallett S, Deeks JJ, Reitsma JB, et al. QUADAS-2: a revised tool for the quality assessment of diagnostic accuracy studies. Ann Intern Med. 2011 Oct 18;155(8):529-36..

12 Rutter CM, Gatsonis CA. A hierarchical regression approach to meta-analysis of diagnostic test accuracy evaluations. Stat Med. 2001 Oct 15;20(19):2865-84.. 


\section{Cochrane Abstract}

Background: Late-onset infection is the most common serious complication associated with hospital care for newborn infants. Because confirming the diagnosis by microbiological culture typically takes 24-48 h, the serum level of the inflammatory marker C-reactive protein (CRP) measured as part of the initial investigation is used as an adjunctive rapid test to guide management in infants with suspected late-onset infection. Objectives: The aim of this study was to determine the diagnostic accuracy of serum CRP measurement in detecting late-onset infection in newborn infants. Search Methods: We searched electronic databases (MEDLINE, Embase, and Science Citation Index to September 2017), conference proceedings, previous reviews, and the reference lists of retrieved articles. Selection Criteria: We included cohort and cross-sectional studies evaluating the diagnostic accuracy of serum CRP levels for the detection of late-onset infection (occurring more than $72 \mathrm{~h}$ after birth) in newborn infants. Data Collection and Analysis: Two review authors independently assessed eligibility for inclusion, evaluated the methodological quality of included studies, and extracted data to estimate diagnostic accuracy using hierarchical summary receiver operating characteristic models. We assessed heterogeneity by examining variability of study estimates and overlap of the $95 \%$ confidence interval $(\mathrm{Cl})$ in forest plots of sensitivity and specificity. Main Results: The search identified 20 studies (1,615 infants). Most were small, single-center, prospective cohort studies conducted in neonatal units in high- or middle-income countries since the late 1990s. Risk of bias in the included studies was generally low with independent assessment of index and reference tests. Most studies used a prespecified serum CRP threshold level as the definition of a "positive" index test (typical cutoff level between 5 and 10 $\mathrm{mg} / \mathrm{L}$ ) and the culture of a pathogenic microorganism from blood as the reference standard. At median specificity (0.74), sensitivity was 0.62 (95\% Cl $0.50-0.73)$. Heterogeneity was evident in the forest plots, but it was not possible to conduct subgroup or meta-regression analyses by gestational ages, types of infection, or types of infecting microorganism. Covariates for whether studies used a predefined threshold or not, and whether studies used a standard threshold of between 5 and $10 \mathrm{mg} / \mathrm{L}$, were not statistically significant. $\mathbf{A u}$ thors' Conclusions: The serum CRP level at initial evaluation of an infant with suspected late-onset infection is unlikely to be considered sufficiently accurate to aid early diagnosis or select infants to undergo further investigation or treatment with antimicrobial therapy or other interventions. 